\title{
1.5. Anorexia Nervosa in Males: Similarities and Differences to Anorexia Neroosa in Females
}

\author{
Arthur Crisp* and Collaborators ${ }^{\dagger}$
}

Keywords: anorexia nervosa; males; females; comparisons

\section{INTRODUCTION}

Previous single case reports of males with anorexia nervosa from our database focused on the roles of gender and general personal identity conflicts and panic/guilt over the consequences of impulsivity in precipitating out the illness (Davis \& Crisp, 1993; Fry \& Crisp, 1989; Toms \& Crisp, 1972), the growth stunting but reversible effects of early onset (Toms \& Crisp, 1972), and a 35-year long follow-up of a case first seen in 1959 (Crisp, 1995).

Our first reported series of 13 male patients (Crisp \& Toms, 1972) drew attention to the many similarities to a female population but emphasised, descriptively, the high prevalence of psychiatric morbidity in the parents, often bound in with phobic avoidance behaviours and commonly coupled with over-protectiveness of the patient during childhood.

A study of the hormonal status of 12 male patients as their body weights were restored to normal levels (Crisp, Hsu, Chen, \& Wheeler 1982; Wheeler, Crisp, Hsu, \& Chen 1983) revealed that, as with females, at a low weight there was no LH response to releasing hormone stimulation. As weight was gained to the high $40 \mathrm{kgs}$ range, $\mathrm{LH}$ responses were enhanced. On reaching target weights (over $60 \mathrm{kgs}$ ) the response tended to normalise. Meanwhile, testosterone levels rose linearly in relation to weight gained. The important psychotherapeutic implications of the accompanying psychopathological changes driven by these somatic changes were highlighted.

Subsequent clinical reports on 36 male patients, included a comparative study with a series of 100 female cases (Crisp, Burns, \& Bhat, 1986) and a follow-up study of 27 of them for a mean length of eight

* Correspondence to: Professor Arthur Crisp, 113 Copse Hill, London SW20 0NT, UK. E-mail: acrisp@sgul.ac.uk

${ }^{\dagger}$ See pages $142-143$. years (Burns \& Crisp 1984). These studies again revealed many similarities between male and female cases, e.g. only minor differences in social class background, mean ages of onset and presentation, degree of premorbid overweight. Dietary patterns were also generally similar except that the females more frequently ingested large quantities of laxatives. With parents, weight disorders were even more common than within the female series. Thus, within the 105 females low body weight of anorectic proportions was present in 14 mothers, nine fathers and seven siblings. Three of the mothers had current anorexia nervosa. Major obesity was present in 10 instances, most often the mother. So far as the 36 males were concerned, one father and four mothers were recorded as having had definite anorexia nervosa; this was also the case for a number of siblings. Ten of the fathers and seven of the mothers were markedly obese. In one family both parents had had definite anorexia nervosa.

The follow-up studies revealed somewhat different predictors of outcome between the sexes. For males, absence of premorbid sexual activity boded ill. Moreover, greater impulsivity, including buli$\mathrm{mia} /$ vomiting within the illness, was not predictive of poor outcome, in contrast to the females. Thus, whilst the ascetic female anorectic did better, in the long term, than her impulse ridden counterpart, this was not so in the male series.

In the male series, gender identity doubt, sometimes coupled with panic recognised as being due to potential impulsivity, seemed to remain a major precipitating factor, with the anorectic regression to pre-puberty forging the defence. However, it could be that such 'gender doubt' was also a common, though sometimes less emphasised, premorbid feature in the female anorectic.

More recently, and related to other studies of patterns within the illness over the last 35 years (see this 
Issue, Paper 2), the males in our series have shown unchanging age of onset (Hindler, Crisp, McGuigan, \& Joughin, 1994) and mean levels of maximum-ever (i.e. premorbid) body weights and lowest-ever weights within the illness, prior to presentation (Figure 1). Over the same period, mean body heights of males within the data-set have likewise not changed (Joughin, Varsou, Gowers, \& Crisp, 1992).

\section{PRESENT REPORT}

This reflects our wish to explore whether some of these earlier findings could be confirmed within the overall data set (1960-1995). This comprises 751 females and 62 males presenting with anorexia nervosa and 176 females and 17 males presenting with some other eating disorder and with a confirmed past history of anorexia nervosa (See this Issue, Introduction).

Eighty-eight per cent of men and $78 \%$ of women were single. The trend towards parental social class (Registrar General's Classification of Occupation) difference persists, with just half of the males coming from Social Class 1 or 2 backgrounds, compared with two thirds of the females. The mean age of onset of initial attempts at dieting (19.0 years for males cf. 17.4 years for females) and the later age of onset of the illness (onset of severe restriction of calorie intake) in the males is noteworthy though neither difference quite reached statistical significance. Tables $1 \mathrm{a}$ and $1 \mathrm{~b}$ show some other such key characteristics of the two populations. There are no significant differences except for those governed by differences in body height. Thus BMI characteristics preceding and within the illness are again similar, with mean lowest BMI within the illness up until time of presentation signalling the severity of the disorder for all these patients. This contrasts with differences between the mean reported preferred weights (transmuted into preferred BMIs) for the two populations (18.6 for males and 17.1 for females).

Whereas there were no differences in the percentages of males and females reporting the main dietary patterns of abstinence or bulimia and vomiting, there were tendencies (Table 2) for laxative abuse to be more common amongst females and for vegetarianism to be more common in males. This translated to a significant difference in males for veganism $(p=0.01)$ and alcohol abuse $(p=0.01)$. Females were also significantly much more likely to hoard food. There were no differences in respect of frequency

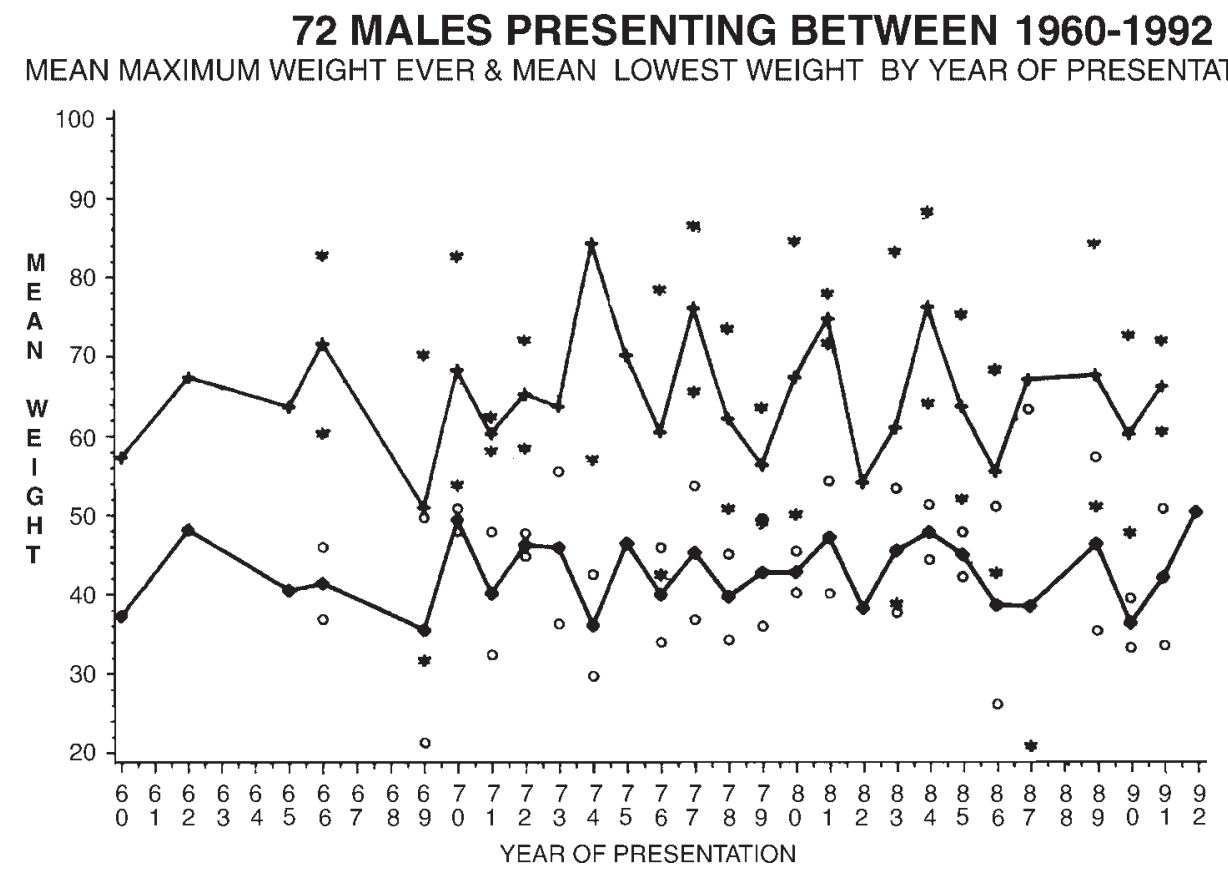

Figure 1. Seventy-two males presenting between 1960-1992. Mean maximum weight ever and mean lowest weight by year of presentation 
Table 1a. Anorexia nervosa at presentation $(n=813)$

\begin{tabular}{lcrc}
\hline & Female $(n=751)$ & Male $(n=62)$ & $p$-value \\
\hline Mean age at onset (years) & $18.5 \pm 5.5$ & $20.1 \pm 8.0$ & n.s. \\
Mean age at presentation (years) & $23.7 \pm 8.2$ & $24.4 \pm 8.9$ & n.s. \\
Mean duration of illness at presentation (years) & $4.9 \pm 6.3$ & $4.1 \pm 4.4$ & n.s. \\
Mean body weight at presentation (kgs) & $39.4 \pm 5.6$ & $45.6 \pm 7.7$ & $<0.0001$ \\
Mean body height at presentation (cms) & $161.8 \pm 6.9$ & $172.1 \pm 9.0$ & $<0.0007$ \\
Mean \% MMPW at presentation & $70.9 \pm 8.4$ & $72.1 \pm 7.9$ & n.s. \\
Mean BMI at presentation & $15.0 \pm 1.7$ & $15.3 \pm 1.7$ & n.s. \\
Mean lowest weight so far, within illness (kgs) & $36.0 \pm 5.9$ & $42.1 \pm 7.3$ & $<0.0000$ \\
Mean lowest \% MMPW so far, within illness & $64.7 \pm 9.2$ & $66.8 \pm 8.5$ & n.s. \\
Mean lowest BMI so far, within illness & $13.7 \pm 1.8$ & $14.2 \pm 1.8$ & n.s. \\
\hline
\end{tabular}

The St George's Anorexia Nervosa database 1960-1995 (1006 cases). Some clinical characteristics of 813 patients presenting with the disorder. Body weights (including indoor clothing/shoeless) and heights measured at presentation. Lowest weight ever, within the illness, if earlier in time, is usually a reported measured weight (sources of such information usually included all of (1) referring physician (2) patient (3) parent(s), (4) spouse, if married)

of usual/severe diet related rituals or of excessive commitment to exercise.

Eighty per cent of males and $77 \%$ of females had parents living together. Mean parental ages at time of probands' presentations were nearly identical for the sexes (mothers: males 53.0 years, females 52.5 years; fathers: males 56.7 years, females 55.8 years). Thirty six per cent of male and $46 \%$ of female patients described their parents' relationship as poor. The family dynamics were rated by the assessor as overprotective in $76 \%$ of male and $62 \%$ of female cases $(p=0.04)$ and enmeshed in $84 \%$ of male and $70 \%$ of female cases $(p=0.02)$. In respect of rated impoverishment of parental relationship and family conflict avoidance patterns, there were no differences. Maternal psychosis (schizophrenia or psychotic affective disorder) was recorded as present in $6 \%$ of male and $1 \%$ of female patients. Regarding history of or present eating disorders and related behaviours in the parents the findings of our earlier report of 105 female patients (Crisp, Hsu, Harding, \& Hartshorn, 1980) were mostly borne out. Thus, Table 3 reveals the much greater likelihood of anorexia nervosa in the mother (judged fairly certainly to have occurred in the past and/or be evident at presentation of the parents) for both male and female cases; also high levels of excessive concern about and behaviour directed at strict maintenance of normal body weight. Obesity (moderate/severe) was rated as present in a quarter of all mothers. Similarly, for fathers, anorexia nervosa was present in one male case and $5 \%$ of female cases. Fathers of male cases were

Table 1b. Past history of anorexia nervosa; other eating disorder at presentation $(n=193)$

\begin{tabular}{lccc}
\hline & Female $(n=176)$ & Male $(n=17)$ & $p$-value \\
\hline Mean age at onset of eating disorder (years) & $17.7 \pm 4.0$ & $17.9 \pm 4.8$ & n.s. \\
Mean age at presentation (years) & $23.5 \pm 7.8$ & $21.9 \pm 6.6$ & n.s. \\
Mean weight at presentation (kgs) & $52.1 \pm 6.6$ & $55.3 \pm 9.3$ & n.s. \\
Mean height at presentation (cms) & $162.4 \pm 6.9$ & $171.3 \pm 12.7$ & $<0.01$ \\
Mean \% MMPW at presentation & $93.3 \pm 9.6$ & $90.7 \pm 8.7$ & n.s. \\
Mean BMI at presentation & $19.7 \pm 2.1$ & $43.6 \pm 1.8$ & n.s. \\
Mean lowest weight so far, within illness (kgs) & $40.2 \pm 5.5$ & $71.0 \pm 9.7$ & n.s. \\
Mean lowest \% MMPW so far, within illness & $72.1 \pm 8.5$ & $14.6 \pm 2.1$ & n.s. \\
Mean lowest BMI so far, within illness & $15.2 \pm 2.0$ & n.s. \\
\hline
\end{tabular}

The St George's Anorexia Nervosa database 1960-1995 (1006 cases). Some clinical characteristics of 193 presenting with some other eating disorder but with a clear history of past anorexia nervosa within the illness. Body weights (including indoor clothing/shoeless) and heights measured at presentation. Lowest weight ever, within the illness, if earlier in time, is usually a reported measured weight (sources of such information usually included all of (1) referring physician (2) patient (3) parent(s), (4) spouse, if married) 
Table 2. Some clinical characteristics (\% rated 'usual' /'severe' in contrast to 'absent' /'occasional')

\begin{tabular}{lcccc}
\hline & \multicolumn{2}{c}{ At presentation } & \multicolumn{2}{c}{ Ever, within the illness } \\
\cline { 2 - 4 } & Males & Females & Males & Females \\
\cline { 2 - 4 } & 8 & 15 & 15 & 21 \\
Bulimia & 21 & 22 & 23 & 30 \\
Vomiting & 15 & 26 & 43 & 33 \\
Laxative abuse & 43 & 35 & 0 & 37 \\
Vegetarianism & 9 & 2 & 22 & 11 \\
Veganism & 0 & 8 & 80 & 70 \\
Hoarding food & 13 & 53 & 70 & 69 \\
Alcohol abuse & 60 & 54 & 63 \\
Ritualistic activity & 54 & & & 3 \\
Excessive exercise & &
\end{tabular}

significantly more often obese $(p=0.02)$. Alcohol addiction was judged to be present in $3-5 \%$ of mothers and $14 \%$ of fathers.

\section{CONCLUSIONS}

In nearly all respects anorexia nervosa is the same in male and female subjects. The disorder sadly and radically diminishes the differences between the sexes, more so than is the case with any other functional mental illness. However there are certain clinical differences that may shed light on the condition. Age of male onset is later than age of female onset within this largely Caucasian population. This can be set alongside the finding that age of onset is earlier amongst non-white (Asian/ Afrocarribean/Chinese) female anorectics (Soomro, Crisp, Lynch, Tran, \& Joughin, 1995). In both instances this relates to differences in growth rate (between the sexes and the different racial groups). It hints again at the close link between the disorder and puberty about which it pivots. Males appear to come significantly more often (though still not frequently so) from a severely psychiatrically disturbed parental background, though conflict avoidant parental behaviour patterns (e.g. agoraphobia, alcohol abuse, depression) are common amongst the parents for both sexes.
Family over-protectiveness towards and enmeshment with the patient as a child are judged to have been more common features in the male, as is paternal obesity.

Gender identity doubt is one seedbed for the condition, if distress related to it becomes construed by the patient as related to body shape and fatness, and it was quite common amongst males in this series. Panic about possible homosexuality prompting anorexia nervosa as a defence continued to be a fairly common finding in the clinic over the last decade and a half. One notable distinguishing feature was the difference in reported ideal BMI between males and females. Throughout the life of the clinic, attention has been paid to ideal weights for height as expressed by both male and female patients. For female patients of average height this ideal and maximum desirable weight is nearly always just above 7 stones ( $44.5 \mathrm{kgs}$ ). Over the years we have also shown the threshold significance of this kind of weight both in relation to endocrinological status and to disappearance of female body fat. Male body fat and female body fat are very differently distributed. Within male puberty the emphasis is on lean body mass and, with growth being slower, a greater ultimate height is achieved. In the female, body fat deposited within puberty is essentially reproductive in its purpose and puberty concludes

Table 3. Some anorexia nervosa related behaviours/phenomena, in parents

\begin{tabular}{lcccrr}
\hline & \multicolumn{2}{c}{$\%$ Mothers } & & \multicolumn{2}{c}{$\%$ Fathers } \\
\cline { 2 - 3 } & Males & Females & & Males & Females \\
\hline Anorexia Nervosa & 14 & 10 & 1 & 12 \\
Excessive normal body weight concerns & 25 & 20 & 23 & 12 & 13 \\
Obesity & 22 & 5 & & 14 & 14 \\
Alcohol abuse & 3 & 5 & & 14 & 14 \\
\hline
\end{tabular}


with a biological statement of ripeness to reproduce. If the personal perception of fatness as an aspect of body shape is the only factor determining anorexia nervosa coupled with the quest to rid the body of it, the male, who traditionally at the end of puberty has only about $12 \%$ of his body as fat, has less distance to travel in this respect than the female (whose body fat comprises about $25 \%$ of her body weight by this time). Of course other excesses of fat may be superimposed, especially these days. Our clinical view is that elimination of such fatness in the female is simply a marker of the profound psychobiological changes that accompany it and which provide her with the haven she needs. However, these differences between the male and the female may be important in terms of incidence, prevalence and outcome.

So far as outcome in the present populations is concerned, this depended to some extent on the sex of the individual. Thus, having been sexually active prior to illness-onset carried a better prognosis for males, as did greater impulsivity, in the form of bulimia, within it. If these differences are confirmed in other male/female anorectic populationstthen the finding may add to the important and doubtless vigorous debate about cultural aspects of the background to eating disorders.

\section{REFERENCES}

Burns, T., \& Crisp, A. H. (1984). Outcome of anorexia nervosa in males. British Journal of Psychiatry, 145, 319325.

Crisp, A. H. (1995). Anorexia nervosa in a young male. In J. Werne (Ed.), Treating eating disorders (pp. 1-30). San Francisco: Jossey Bass.
Crisp, A. H., Burns, T., \& Bhat, A. V. (1986). Primary anorexia nervosa in the male and female: A comparison of clinical features and prognosis. British Journal of Medical Psychology, 59, 123-132.

Crisp, A. H., Hsu, L. K. G., Chen, C. N., \& Wheeler, M. (1982). Reproductive hormone profiles in male anorexia nervosa before, during and after restoration of body weight to normal: A study of 12 patients. International Journal of Eating Disorders, 1, 3-9.

Crisp, A. H., Hsu, L. K. G., Harding, B., \& Hartshorn, J. (1980). Clinical features of anorexia nervosa: A study of a consecutive series of 102 female patients. Journal of Psychosomatic Research, 24, 179-191.

Crisp, A. H., \& Toms, D. A. (1972). Primary anorexia nervosa or weight phobia in the male: Report on 13 cases. British Medical Journal, 1, 334-338.

Davis, J. da Rosa \& Crisp, A. H. (1993). Post-traumatic anorexia nervosa-A case study. British Journal of Medical Psychology, 66, 79-87.

Fry, R., \& Crisp, A. H. (1989). Adoption and identity: A case of anorexia nervosa. British Journal of Medical Psychology, 62, 143-152.

Hindler, C. G., Crisp, A. H., McGuigan, S., \& Joughin, N. (1994). Anorexia nervosa: change over time in age of onset, presentation and duration of illness. Psychological Medicine, 24, 719-729.

Joughin, N., Varsou, E., Gowers, S, \& Crisp, A. H. (1992). Relative tallness in anorexia nervosa. International Journal of Eating Disorders, 12, 193-207.

Soomro, G. M., Crisp, A. H., Lynch, D., Tran, D., \& Joughin, N. (1995). Anorexia nervosa in 'non-white' populations. British Journal of Psychiatry, 167, 385389.

Toms, D. A., \& Crisp, A. H. (1972). Weight phobia in an adolescent male with stunted development. Journal of Psychosomatic Research, 16, 289-295.

Wheeler, M. J., Crisp, A. H., Hsu, L. K. G., \& Chen, C. N. (1983). Reproductive hormone changes during weight gain in male anorectics. Clinical Endocrinology, 18, 423429. 